Review article

\title{
Neuron-microglia interaction by purinergic signaling in neuropathic pain following neurodegeneration
}

Makoto Tsuda ${ }^{1}$, Kazuhide Inoue ${ }^{2}$

${ }^{1}$ Department of Life Innovation, ${ }^{2}$ Department of Molecular and System Pharmacology, Graduate School of Pharmaceutical Sciences, Kyushu University, Fukuoka, Japan

*Corresponding authors:

Makoto Tsuda, Ph.D.

3-1-1 Maidashi, Higashi-ku, Fukuoka 812-8582, Japan

Tel. \& Fax.: 81-92-642-6628

E-mail: tsuda@phar.kyushu-u.ac.jp

Highlights

- Spinal microglia increase purinergic receptors following nerve damage.

- Microglial purinergic receptors are crucial for neuropathic pain.

- Hyperexcitability in dorsal horn neurons involves microglial purinergic signaling.

- Microglial purinergic receptors might be promising targets for treating neuropathic pain. 


\begin{abstract}
Neuropathic pain, a chronic pain condition following nerve damage and degeneration, involves aberrant excitability in the dorsal horn of the spinal cord. A growing body of evidence has shown that the aberrant excitability might not be a consequence merely of changes in neurons, but rather of multiple alterations in glial cells, such as microglia, the immune cells of the central nervous system. Extracellular nucleotides play an important role in neuron-microglia communication through purinergic $\mathrm{P} 2 \mathrm{X}$ and $\mathrm{P} 2 \mathrm{Y}$ receptors expressed in microglia. Importantly, inhibiting the function or expression of these microglial molecules suppresses aberrant excitability of dorsal horn neurons and neuropathic pain, suggesting a crucial role for microglial purinergic signaling in mechanisms of neuropathic pain. Here, we describe recent advances in the understanding of neuron-microglia interactions by purinergic signaling in neuropathic pain following neurodegeneration.
\end{abstract}

Keywords: neuropathic pain; microglia; purinergic receptors; transcription factors; proinflammatory cytokines; chemokines; spinal cord 


\section{Introduction}

We experience acute pain in response to noxious stimuli, which plays an important role as an early warning device that alerts us to the presence of damaging stimuli. Even if tissue damage is unavoidable and leads to heightened pain sensitivity in the inflamed and surrounding tissue (inflammatory pain), pain still has physiological significance. For example, pain might assist in wound repair, because contact with the damaged area is often minimized until healing has occurred. However, neuropathic pain, which occurs after nerve damage and degeneration induced by infection, autoimmune disease, or traumatic nerve injury, is a type of pathological pain that does not resolve even after the overt tissue damage has healed. This chronic pain provides no known physiological advantage, because it does not signal tissue damage. Symptoms of neuropathic pain are spontaneous pain, hyperalgesia (increased pain perception of noxious stimuli), and tactile allodynia (pain hypersensitivity to normally innocuous stimuli). Neuropathic pain, especially allodynia, is frequently resistant to currently available drugs when administered at doses that do not produce significant side effects. We are now beginning to understand that neuropathic pain is not just a symptom of disease, but is a consequence of disordered functioning of the nervous system (Beggs et al., 2012; Costigan et al., 2009).

Accumulating evidence demonstrating how peripheral nerve damage creates neuropathic pain has indicated that molecular and cellular alterations in primary sensory neurons and in the spinal dorsal horn (SDH) after peripheral nerve injury (PNI) have important roles in the pathogenesis of neuropathic pain (Scholz and Woolf, 2002; Woolf and Mannion, 1999; Woolf and Salter, 2000). A rapidly growing body of evidence indicates that spinal glial cells, in particular microglia, play a critical role in the pathogenesis of neuropathic pain. Extracellular nucleotides play an important role in neuron-glia communication through purinergic $\mathrm{P} 2 \mathrm{X}$ and $\mathrm{P} 2 \mathrm{Y}$ receptors $(\mathrm{P} 2 \mathrm{XR}$ and $\mathrm{P} 2 \mathrm{YR}$, respectively). Microglia, which are thought to be residential macrophages in the central nervous system (CNS), express P2XRs and P2YRs, mainly P2X4R and P2X7R, as well as P2Y2R, P2Y6R, and P2Y12R (Inoue, 2006). Results from animal models of neuropathic pain have shown that microglial purinergic signaling via $\mathrm{P} 2 \mathrm{X} / \mathrm{YRs}$ is crucial for pathologically modulating pain processing in the SDH after PNI and for PNI-induced pain hypersensitivity (Beggs et al., 2012; Tsuda et al., 2005; Tsuda et al., 2013c). In this review article, we describe recent advances in our understanding of neuron-microglia interactions by purinergic signaling in neuropathic pain following neurodegeneration. 


\section{Microglia in the SDH}

Microglial cells are known as resident tissue macrophages in the CNS and constitute $5-10 \%$ of total cells in the adult CNS. Accumulating evidence indicates that the origin of microglia might be primitive macrophages in the yolk sac. Fate mapping has revealed that microglia arise from early yolk sac-derived precursors that leave the yolk sac on E8.5-E9.0, migrating to the neuroectoderm via the primitive blood stream (Ginhoux et al., 2010). The precursors have erythro-myeloid potential (Kierdorf et al., 2013). Microglial generation is dependent on the transcription factors PU.1 and interferon regulatory factor 8 (IRF8) (Kierdorf et al., 2013), as well as interleukin-34 (IL-34) (Greter et al., 2012; Wang et al., 2012). However, microglial generation is independent of Myb (Schulz et al., 2012), which is essential for bone marrow-derived macrophages (Hashimoto et al., 2013; Yona et al., 2013). The yolk-sac-derived microglia presumably remain throughout life and might be maintained by self-renewal in the healthy CNS with little contribution from bone-marrow-derived monocytes/macrophages (Ajami et al., 2007).

In the adult, microglia have small cell bodies bearing branched and motile processes, which might monitor the local environment in the CNS (Davalos et al., 2005; Nimmerjahn et al., 2005). Microglia rapidly respond to a wide range of stimuli that threaten physiological homeostasis, including PNI. In a growing body of literature, it is evident that PNI leads to dramatic activation of microglia in the SDH. This response is commonly observed among various models of neuropathic pain. The morphological features of microglial activation include cell body hypertrophy with thickened and retracted processes, increased cell number, and increased staining of microglial markers, such as CD11b and ionized calcium-binding adapter molecule-1 (Iba1).

A neuronally derived signaling molecule that might be important for microglial activation remains to be determined, but several candidates have been reported. These include monocyte chemoattractant protein-1 (MCP-1 or CCL2) and metalloproteinase-9 (MMP-9), whose expressions are markedly increased in dorsal root ganglion neurons after PNI (Kawasaki et al., 2008a; Tanaka et al., 2004; Thacker et al., 2009; White et al., 2007; Zhang and De Koninck, 2006). Mice lacking chemotactic cytokine receptor 2, a receptor for MCP-1, or MMP-9-deficient mice show reduced microglia activation caused by PNI (Kawasaki et al., 2008a; Zhang et al., 2007). Conversely, intrathecal administration of MCP-1 or MMP-9 into normal rats resulted in microglial activation (Kawasaki et al., 2008a; Thacker et al., 2009). Substrates of MMP-9 for microglial activation remain unclear, but fractalkine, IL- $1 \beta$, and tumor necrosis factor- $\alpha$ (TNF $\alpha)$ could be potential candidates (Suter et al., 2007). 
The number of microglia in the SDH is markedly increased after PNI (Tsuda et al., 2013a). This might be associated with proliferation of resident microglia. Indeed, PNI induced an early and transient increase in the number of microglia positive to proliferation markers, such as bromodeoxyuridine (BrdU), a thymidine analog incorporated into DNA during the S phase of cell cycle (Echeverry et al., 2008; Gehrmann and Banati, 1995; Liu et al., 2000; Narita et al., 2006; Suter et al., 2007; Zhang et al., 2007). The proliferation activity of microglia peaks around 2 days after PNI and then declines to basal levels (Echeverry et al., 2008; Gehrmann and Banati, 1995). Conversely, it was shown that bone marrow-derived cells injected intravenously into lethally irradiated recipient mice infiltrate the SDH parenchyma ipsilateral to the PNI and are positive for Ibal and display microglia-like morphology. However, the ability of bone marrow-derived cells to migrate into the parenchyma of the CNS, including the spinal cord, remains controversial as a result of experimental manipulations, such as irradiation (which could influence the blood-spinal cord barrier) and exogenously injected donor cells (Ajami et al., 2007).

\section{Traumatic nerve injury}

\section{2-1. P2X4Rs}

Activated microglia show dramatic changes in the expression of a variety of genes. Expression of P2X4Rs has been shown to increase in microglia after PNI. The pharmacological blockade of spinal P2X4Rs reverses PNI-induced tactile allodynia (Tsuda et al., 2003), indicating that PNI-induced pain hypersensitivity depends on ongoing purinergic signaling through microglial P2X4Rs. Prevention of PNI-induced allodynia in both P2X4R-knockdown (KD) and knockout $(\mathrm{KO})$ mice demonstrates the necessity of P2X4Rs (Tsuda et al., 2009a; Tsuda et al., 2003; Ulmann et al., 2008). Intrathecal delivery of $\mathrm{P} 2 \mathrm{X} 4 \mathrm{R}$-stimulated microglia induces normal rats to produce allodynia, indicating a P2X4R sufficiency (Tsuda et al., 2005; Tsuda et al., 2003). It was further demonstrated that activation of microglial P2X4Rs leads to a release of brain-derived neurotrophic factor (BDNF) (Trang et al., 2009; Ulmann et al., 2008). BDNF then subsequently induces an altered transmembrane anion gradient in a subpopulation of lamina I neurons in the SDH by downregulating the neuronal chloride transporter, KCC2 (Coull et al., 2005). This change induces changes in $\gamma$-aminobutyric acid and glycine that depolarizes neurons (Coull et al., 2005). Furthermore, local spinal administration of ATP-stimulated microglia alters the phenotype of in vivo spinal lamina I output neurons, such that they relay innocuous mechanical input (Keller et al., 2007). Thus, P2X4R-stimulated microglia release BDNF, which signals lamina I neurons, 
causing aberrant nociceptive output that contributes to neuropathic pain (Beggs et al., 2012; Tsuda et al., 2013c). However, the microglial pain processing involving P2X4R and BDNF after PNI may be essential only in male mice, because upregulation of spinal P2X4Rs after PNI was not observed in female mice and because pharmacological blockade of spinal P2X4Rs did not produce an anti-allodynic effect (Sorge et al., 2015). Furthermore, in female mice, conditional knockout of BDNF in microglia did not suppress PNI-induced allodynia.

Several studies have identified molecules that upregulate P2X4R expression in microglia (Tsuda et al., 2008a; Tsuda et al., 2009b; Tsuda et al., 2008b). Recently, it was found that IRF8 is a crucial transcription factor for P2X4R upregulation (Masuda et al., 2012). IRF8 is a member of the IRF family (IRF1-9) and is expressed in immune cells, such as lymphocytes and dendritic cells (Tamura et al., 2008). Within the spinal cord, IRF8 expression is selectively upregulated in microglia after PNI (Masuda et al., 2012). Additionally, IRF8-KO mice display reduced tactile allodynia after PNI. Furthermore, suppressing spinal IRF8 expression reverses the PNI-induced allodynia, indicating that microglial IRF8 is necessary for allodynia development and maintenance. In in vitro studies using microglial cells, IRF8 promotes expression of P2X4R and other genes associated with reactive states, and PNI-induced P2X4R upregulation is prevented in IRF8-KO mice. Moreover, IRF5 was identified as a target of IRF8 in microglia (Masuda et al., 2014) in other cells (Kurotaki et al., 2013). IRF5 expression is also selectively induced in spinal microglia after PNI in an IRF8-dependent manner. IRF5 is known to predominantly reside in the cytoplasm, and during stimulation-dependent activation, it translocates to the nucleus to activate gene transcription (Tamura et al., 2008). IRF5 protein accumulates in the nuclear fraction when cells are stimulated with fibronectin (Masuda et al., 2014). Interestingly, IRF5 might then directly binds to the promoter region of the P2rx4 gene and lead to an induction of P2X4R expression in microglial cells. Indeed, IRF5-KO mice demonstrate a reduction in spinal P2X4R upregulation and allodynia after PNI (Masuda et al., 2014). Therefore, it is thought that an IRF8-IRF5 transcriptional cascade could contribute to the shift of spinal microglia towards a P2X4R-expressing reactive state after PNI (Tsuda et al., 2013b, c).

It has been shown that a large amount of $\mathrm{P} 2 \mathrm{X} 4 \mathrm{R}$ protein within microglia localizes to intracellular lysosomes (Qureshi et al., 2007), and P2X4R protein remains stable within the proteolytic environment. How P2X4R protein is recruited to the cell surface of microglia for detecting extracellular ATP remains elusive, although recent studies have shown that trafficking of $\mathrm{P} 2 \mathrm{X} 4 \mathrm{R}$ protein to the cell surface occurs when microglia are stimulated by a toll-like receptor 4 agonist, lipopolysaccharide (Boumechache et al., 
2009; Toulme et al., 2010), or the $\mathrm{Ca}^{2+}$ ionophore ionomycin (Qureshi et al., 2007). Furthermore, it was found that the chemokine CCL2 increases P2X4R protein levels on the cell surface (without changing total cellular expression) through CCR2 (Toyomitsu et al., 2012). Single-molecule imaging to track P2X4Rs in the processes of microglia showed that lateral mobility of P2X4Rs is enhanced in activated microglia by $\mathrm{p} 38$, a member of the MAPKs pathway (Toulme and Khakh, 2012). Previous reports have also shown that p38 is activated in spinal microglia after PNI and contributes to neuropathic pain (Jin et al., 2003; Tsuda et al., 2004). Thus, post-translational regulation to enhance P2X4R expression and mobility on the cell surfaces might render microglia hyperresponsive to extracellular ATP, which might be important in neuropathic pain.

\section{2-2. P2X7R}

In P2X7R-KO mice, PNI-induced mechanical hypersensitivities are reduced (Chessell et al., 2005), indicating the involvement of P2X7Rs in neuropathic pain. This was supported by findings showing that systemic administration of A-740003 and A-438079, selective inhibitors of P2X7R, reduces tactile allodynia in three different rat models of neuropathic pain (Honore et al., 2006). Furthermore, intrathecal administration of A-438079 attenuates the development of mechanical hypersensitivity. The predominant type of cells expressing these receptors in the SDH after PNI might be microglia, because P2X7R mRNA and protein were localized in microglia in the SDH (Kobayashi et al., 2011). In another model, intrathecal administration of brilliant blue G, which can block P2X7R, reduces both behavioral hypersensitivity and enhancement of CD11b staining levels in the SDH (He et al., 2012). It was also shown that intravenous administration of A-438079 reduces innocuous stimuli-evoked activity of SDH neurons in neuropathic rats, suggesting that the activation of microglial P2X7Rs might influence neuronal excitation of SDH neurons (McGaraughty et al., 2007). P2X7R is one of the key players in the release of proinflammatory cytokines, such as IL-1 $\beta$, IL-6, and TNF $\alpha$, from activated microglia (Ferrari et al., 1997; Hide et al., 2000; Shigemoto-Mogami et al., 2001). In the SDH, IL-1 $\beta$ is released from LPS-primed microglia following ATP stimulation in a P2X7R-dependent manner (Clark et al., 2010a). These proinflammatory cytokines potently modulate the activity of excitatory and inhibitory SDH neurons (Ikeda et al., 2007; Kawasaki et al., 2008b). In microglial cells pretreated with LPS, the activation of P2X7Rs induces release of cathepsin S (Clark et al., 2010b), a lysosomal cysteine protease that contributes to neuropathic pain (Clark et al., 2007). Furthermore, it has been shown that microglial P2X7R stimulation can induce synthesis and release of the chemokines CCL3 and CXCL2 (Kataoka et al., 2009; Shiratori et al., 2010). 
CCL3 expression in the SDH increases after PNI, and intrathecal injection of a neutralizing antibody for CCL3 reduces neuropathic pain behaviors (Kiguchi et al., 2010a; Kiguchi et al., 2010b; Matsushita et al., 2014). Thus, microglial P2X7Rs might participate in the neuronal hyperexcitability of SDH neurons and the development of neuropathic pain through production of proinflammatory cytokines and chemokines.

\section{2-3. P2Y12R}

Within the CNS, P2Y12R is specifically expressed in resident microglia (Haynes et al., 2006; Kobayashi et al., 2006; Pausch et al., 2004; Sasaki et al., 2003). Following PNI, P2Y12R expression levels increase in microglia in the SDH (Kobayashi et al., 2008; Tozaki-Saitoh et al., 2008). Regarding the role of P2Y12Rs in neuropathic pain, it was shown that intrathecal administration of P2Y12R antagonists (MRS2395 and AR-C69931MX) and a P2Y12R antisense oligonucleotide suppress the development of neuropathic pain after PNI (Kobayashi et al., 2008; Tozaki-Saitoh et al., 2008). P2Y12R-KO mice fail to show tactile allodynia (Tozaki-Saitoh et al., 2008).

Additionally, a single administration of AR-C69931MX (intrathecally) and clopidogrel (orally) to PNI rats reverses the established tactile allodynia (Tozaki-Saitoh et al., 2008), indicating on-going activation of this receptor.

Studies have identified P2Y12R as a primary sensor for extracellular ATP in vitro (Honda et al., 2001; Ohsawa et al., 2007) and in vivo (Haynes et al., 2006). Therefore, it is possible that upregulation of microglial P2Y12R may influence the ability of microglia to extend the tips of their branched processes towards neighboring cells that presumably release ATP, and these may in turn develop new microglial connections to other cells. Electron microscopy revealed that SDH microglia adhere to and engulf both injured and uninjured myelinated axons, an interaction that has been rarely observed in the normal SDH (Maeda et al., 2010). The attachment of microglia to myelinated axons is suppressed by AR-C69931MX, suggesting that microglial engulfment might require P2Y12Rs. Thus, it is speculated that engulfment of myelinated axons by activated microglia via P2Y12R signaling in the SDH may be crucial for effective interactions between axons and microglia that induces the pathogenic process leading to neuropathic pain.

\section{Chemotherapeutic agent-induced neuropathic pain}

Neurodegeneration is a serious side effect of many commonly used anti-cancer agents, such as paclitaxel, vincristine, cisplatin, and oxaliplatin. The dose-limiting toxic effects of these chemotherapeutic agents include sensory abnormalities of extremities 
usually occurring in a stocking-and-glove distribution in addition to motor dysfunction in patients and results in a long-term negative impact on patient quality of life (Mantyh, 2006). It was shown that microglia activation occurs in the SDH of rats or mice treated with paclitaxel (Ochi-Ishi et al., 2014; Peters et al., 2007), vincristine (Kiguchi et al., 2010a; Shen et al., 2015), and oxaliplatin (Di Cesare Mannelli et al., 2014). Furthermore, intrathecal administration of minocycline, a reagent that can inhibit microglial activation, attenuates paclitaxel-induced pain hypersensitivity (Cata et al., 2008; Di Cesare Mannelli et al., 2014). Recently, it was found that paclitaxel-treated rats express increased mRNA levels of CCL3 and its receptor CCR5 in the SDH (Ochi-Ishi et al., 2014). Administering a CCL3-neutralizing antibody into the intrathecal space attenuates the development of paclitaxel-induced mechanical allodynia. Paclitaxel also upregulates expression of P2X7Rs in the SDH, which has been implicated in CCL3 release from microglia (Kataoka et al., 2009). Intrathecal administration of the selective P2X7R antagonist A438079 produces a preventive effect on paclitaxel-induced allodynia (Ochi-Ishi et al., 2014), suggesting the contribution of microglial purinergic receptor signaling via P2X7Rs in the SDH to the development of allodynia in paclitaxel-treated animals. Moreover, a single intrathecal administration of either a CCL3-neutralizing antibody or selective P2X7R antagonists reverses the established mechanical allodynia in paclitaxel- and vincristine-treated rats (Honore et al., 2006; Ochi-Ishi et al., 2014). It is, therefore, possible that ongoing signaling via CCL3 (presumably released from P2X7R-stimulating microglia) is also crucial for maintaining paclitaxel-induced pain hypersensitivity. The detailed mechanisms by which CCL3 mediates paclitaxel-induced mechanical allodynia are not clear. The ability of spinal CCL3 to produce allodynia was reported by studies showing that intrathecal administration of CCL3 to naïve animals results in pain hypersensitivity (Kiguchi et al., 2010b; Matsushita et al., 2014). In the SDH, CCR5 is expressed in activated microglia after PNI (Gamo et al., 2008; Matsushita et al., 2014). In addition, there was no change in CCR1 expression (another CCL3 receptor) in the SDH of paclitaxel-treated rats, as well as no effect of a selective CCR1 antagonist on paclitaxel-evoked pain hypersensitivity (Pevida et al., 2013). These results suggest that spinal CCL3 released from microglia in response to activation of P2X7Rs by extracellular ATP (presumably released from neighboring neurons (Jo and Schlichter, 1999) or glial cells (Fam et al., 2000)) further activates microglia via CCR5 in an autocrine manner, which may in turn lead to altered SDH pain processing (Ochi-Ishi et al., 2014).

\section{Other models of neurodegenerative disease}


Multiple sclerosis (MS) is an inflammatory demyelinating disease of the CNS. A common symptom is chronic pain, which affects between $50 \%$ and $80 \%$ of MS patients (Osterberg et al., 2005). However, the underlying mechanisms of neuropathic pain associated with MS are poorly understood. Experimental autoimmune encephalomyelitis (EAE) is an accepted animal model of MS that shares many pathological features observed in MS patients. In an EAE model, where female C57BL/6 mice are immunized with myelin oligodendrocyte glycoprotein ( $\left.\mathrm{MOG}_{35-55}\right)$, robust tactile allodynia and microglial activation in the SDH were induced in the early disease process (Olechowski et al., 2009). Little is known about the role of purinergic signaling in pain hypersensitivity in EAE models, but increased expression of P2X4R mRNA occurs in spinal microglia in a rat model of EAE (Guo and Schluesener, 2005; Vazquez-Villoldo et al., 2014) and in the optic nerve of MS patients (Vazquez-Villoldo et al., 2014), raising the possibility that microglial P2X4Rs may play a role.

Neuropathic pain is also a common symptom of Guillain-Barre syndrome, a human acute inflammatory demyelinating neuropathy caused by an autoimmune attack on the peripheral nervous system (Hughes and Cornblath, 2005). In experimental autoimmune neuritis (EAN) rats, which is a model of acute inflammatory demyelinating polyradiculoneuropathy, the most common subtype of Guillain-Barre syndrome, P2X4R protein in the SDH is selectively upregulated in activated microglia (Zhang et al., 2008). The time course of accumulation of P2X4R-positive microglia in the SDH corresponds with tactile allodynia, indicating that P2X4R might contribute to EAN-induced neuropathic pain. However, whether pharmacological blockade of these receptors alleviates EAN allodynia remains to be determined.

\section{Conclusion}

We have primarily focused on the role of microglial purinergic signaling in neuropathic pain after neurodegeneration. A model of mechanisms underlying microglia-mediated neuropathic pain modulation in the SDH is presented in Figure 1. Importantly, pharmacological, molecular, and genetic manipulations of the function or expression of these microglial molecules substantially influences pain behaviors and hyperexcitability of the SDH pain pathway. Therefore, spinal microglia critically contribute to pathologically enhanced pain processing in the SDH, and microglial purinergic signaling might be a promising target for treating neuropathic pain. It is expected that an increased understanding of the functions of microglial molecules will provide us with exciting insights into pain mechanisms and clues to develop novel therapeutic agents for the management of neuropathic pain. 


\section{Acknowledgments}

This work was supported by grants from the Ministry of Education, Culture, Sports, Science and Technology of Japan (M.T., K.I.), from the Japan Science and Technology Agency (JST) through the Core Research for Evolutional Science and Technology (CREST) program (K.I.), from Takeda Science Foundation (M.T.), from Toray Science Foundation (M.T.). 


\section{References}

Ajami, B., Bennett, J. L., Krieger, C., Tetzlaff, W., Rossi, F. M., 2007. Local

self-renewal can sustain CNS microglia maintenance and function throughout adult life.

Nat Neurosci 10, 1538-1543.

Beggs, S., Trang, T., Salter, M. W., 2012. P2X4R+ microglia drive neuropathic pain.

Nat Neurosci 15, 1068-1073.

Boumechache, M., Masin, M., Edwardson, J. M., Gorecki, D. C., Murrell-Lagnado, R., 2009. Analysis of assembly and trafficking of native P2X4 and P2X7 receptor complexes in rodent immune cells. J Biol Chem 284, 13446-13454.

Cata, J. P., Weng, H. R., Dougherty, P. M., 2008. The effects of thalidomide and minocycline on taxol-induced hyperalgesia in rats. Brain Res 1229, 100-110.

Chessell, I. P., Hatcher, J. P., Bountra, C., Michel, A. D., Hughes, J. P., Green, P., Egerton, J., Murfin, M., Richardson, J., Peck, W. L., Grahames, C. B., Casula, M. A., Yiangou, Y., Birch, R., Anand, P., Buell, G. N., 2005. Disruption of the P2X7 purinoceptor gene abolishes chronic inflammatory and neuropathic pain. Pain 114, 386-396.

Clark, A. K., Staniland, A. A., Marchand, F., Kaan, T. K., McMahon, S. B., Malcangio, M., 2010a. P2X7-dependent release of interleukin-1beta and nociception in the spinal cord following lipopolysaccharide. J Neurosci 30, 573-582.

Clark, A. K., Wodarski, R., Guida, F., Sasso, O., Malcangio, M., 2010b. Cathepsin S release from primary cultured microglia is regulated by the $\mathrm{P} 2 \mathrm{X} 7$ receptor. Glia 58, $1710-1726$.

Clark, A. K., Yip, P. K., Grist, J., Gentry, C., Staniland, A. A., Marchand, F., Dehvari, M., Wotherspoon, G., Winter, J., Ullah, J., Bevan, S., Malcangio, M., 2007. Inhibition of spinal microglial cathepsin $\mathrm{S}$ for the reversal of neuropathic pain. Proc Natl Acad Sci U S A 104, 10655-10660.

Costigan, M., Scholz, J., Woolf, C. J., 2009. Neuropathic pain: a maladaptive response of the nervous system to damage. Annu Rev Neurosci 32, 1-32.

Coull, J. A., Beggs, S., Boudreau, D., Boivin, D., Tsuda, M., Inoue, K., Gravel, C., Salter, M. W., De Koninck, Y., 2005. BDNF from microglia causes the shift in neuronal anion gradient underlying neuropathic pain. Nature 438, 1017-1021.

Davalos, D., Grutzendler, J., Yang, G., Kim, J. V., Zuo, Y., Jung, S., Littman, D. R., Dustin, M. L., Gan, W. B., 2005. ATP mediates rapid microglial response to local brain injury in vivo. Nat Neurosci 8, 752-758.

Di Cesare Mannelli, L., Pacini, A., Micheli, L., Tani, A., Zanardelli, M., Ghelardini, C., 2014. Glial role in oxaliplatin-induced neuropathic pain. Exp Neurol 261, 22-33. 
Echeverry, S., Shi, X. Q., Zhang, J., 2008. Characterization of cell proliferation in rat spinal cord following peripheral nerve injury and the relationship with neuropathic pain. Pain 135, 37-47.

Fam, S. R., Gallagher, C. J., Salter, M. W., 2000. P2Y(1) purinoceptor-mediated Ca(2+) signaling and $\mathrm{Ca}(2+)$ wave propagation in dorsal spinal cord astrocytes. J Neurosci 20, 2800-2808.

Ferrari, D., Chiozzi, P., Falzoni, S., Hanau, S., Di Virgilio, F., 1997. Purinergic modulation of interleukin-1 beta release from microglial cells stimulated with bacterial endotoxin. J Exp Med 185, 579-582.

Gamo, K., Kiryu-Seo, S., Konishi, H., Aoki, S., Matsushima, K., Wada, K., Kiyama, H., 2008. G-protein-coupled receptor screen reveals a role for chemokine receptor CCR5 in suppressing microglial neurotoxicity. J Neurosci 28, 11980-11988.

Gehrmann, J., Banati, R. B., 1995. Microglial turnover in the injured CNS: activated microglia undergo delayed DNA fragmentation following peripheral nerve injury. $\mathbf{J}$ Neuropathol Exp Neurol 54, 680-688.

Ginhoux, F., Greter, M., Leboeuf, M., Nandi, S., See, P., Gokhan, S., Mehler, M. F., Conway, S. J., Ng, L. G., Stanley, E. R., Samokhvalov, I. M., Merad, M., 2010. Fate mapping analysis reveals that adult microglia derive from primitive macrophages. Science 330, 841-845.

Greter, M., Lelios, I., Pelczar, P., Hoeffel, G., Price, J., Leboeuf, M., Kundig, T. M., Frei, K., Ginhoux, F., Merad, M., Becher, B., 2012. Stroma-derived interleukin-34 controls the development and maintenance of langerhans cells and the maintenance of microglia. Immunity 37, 1050-1060.

Guo, L. H., Schluesener, H. J., 2005. Lesional accumulation of P2X(4) receptor(+) macrophages in rat CNS during experimental autoimmune encephalomyelitis.

Neuroscience 134, 199-205.

Hashimoto, D., Chow, A., Noizat, C., Teo, P., Beasley, M. B., Leboeuf, M., Becker, C. D., See, P., Price, J., Lucas, D., Greter, M., Mortha, A., Boyer, S. W., Forsberg, E. C., Tanaka, M., van Rooijen, N., Garcia-Sastre, A., Stanley, E. R., Ginhoux, F., Frenette, P. S., Merad, M., 2013. Tissue-resident macrophages self-maintain locally throughout adult life with minimal contribution from circulating monocytes. Immunity 38, 792-804. Haynes, S. E., Hollopeter, G., Yang, G., Kurpius, D., Dailey, M. E., Gan, W. B., Julius, D., 2006. The P2Y(12) receptor regulates microglial activation by extracellular nucleotides. Nat Neurosci 9, 1512-1519.

He, W. J., Cui, J., Du, L., Zhao, Y. D., Burnstock, G., Zhou, H. D., Ruan, H. Z., 2012. Spinal P2X(7) receptor mediates microglia activation-induced neuropathic pain in the 
sciatic nerve injury rat model. Behavioural Brain Research 226, 163-170.

Hide, I., Tanaka, M., Inoue, A., Nakajima, K., Kohsaka, S., Inoue, K., Nakata, Y., 2000.

Extracellular ATP triggers tumor necrosis factor-alpha release from rat microglia. $\mathrm{J}$

Neurochem 75, 965-972.

Honda, S., Sasaki, Y., Ohsawa, K., Imai, Y., Nakamura, Y., Inoue, K., Kohsaka, S., 2001.

Extracellular ATP or ADP induce chemotaxis of cultured microglia through

Gi/o-coupled P2Y receptors. J Neurosci 21, 1975-1982.

Honore, P., Donnelly-Roberts, D., Namovic, M. T., Hsieh, G., Zhu, C. Z., Mikusa, J. P., Hernandez, G., Zhong, C., Gauvin, D. M., Chandran, P., Harris, R., Medrano, A. P., Carroll, W., Marsh, K., Sullivan, J. P., Faltynek, C. R., Jarvis, M. F., 2006. A-740003 $[\mathrm{N}-(1-\{[($ cyanoimino $)(5-$ quinolinylamino $)$

methyl]amino \}-2,2-dimethylpropyl)-2-(3,4-dimethoxyphenyl)acetamide], a novel and selective P2X7 receptor antagonist, dose-dependently reduces neuropathic pain in the rat. J Pharmacol Exp Ther 319, 1376-1385.

Hughes, R. A., Cornblath, D. R., 2005. Guillain-Barre syndrome. Lancet 366, 1653-1666.

Ikeda, H., Tsuda, M., Inoue, K., Murase, K., 2007. Long-term potentiation of neuronal excitation by neuron-glia interactions in the rat spinal dorsal horn. Eur J Neurosci 25 , 1297-1306.

Inoue, K., 2006. The function of microglia through purinergic receptors: Neuropathic pain and cytokine release. Pharmacol Ther 109, 210-226.

Jin, S. X., Zhuang, Z. Y., Woolf, C. J., Ji, R. R., 2003. p38 mitogen-activated protein kinase is activated after a spinal nerve ligation in spinal cord microglia and dorsal root ganglion neurons and contributes to the generation of neuropathic pain. J Neurosci 23, 4017-4022.

Jo, Y. H., Schlichter, R., 1999. Synaptic corelease of ATP and GABA in cultured spinal neurons. Nat Neurosci 2, 241-245.

Kataoka, A., Tozaki-Saitoh, H., Koga, Y., Tsuda, M., Inoue, K., 2009. Activation of P2X7 receptors induces CCL3 production in microglial cells through transcription factor NFAT. J Neurochem 108, 115-125.

Kawasaki, Y., Xu, Z. Z., Wang, X., Park, J. Y., Zhuang, Z. Y., Tan, P. H., Gao, Y. J., Roy, K., Corfas, G., Lo, E. H., Ji, R. R., 2008a. Distinct roles of matrix metalloproteases in the early- and late-phase development of neuropathic pain. Nature medicine 14, 331-336.

Kawasaki, Y., Zhang, L., Cheng, J. K., Ji, R. R., 2008b. Cytokine mechanisms of central sensitization: distinct and overlapping role of interleukin-1beta, interleukin-6, and tumor 
necrosis factor-alpha in regulating synaptic and neuronal activity in the superficial spinal cord. J Neurosci 28, 5189-5194.

Keller, A. F., Beggs, S., Salter, M. W., De Koninck, Y., 2007. Transformation of the output of spinal lamina I neurons after nerve injury and microglia stimulation underlying neuropathic pain. Molecular pain 3, 27.

Kierdorf, K., Erny, D., Goldmann, T., Sander, V., Schulz, C., Perdiguero, E. G., Wieghofer, P., Heinrich, A., Riemke, P., Holscher, C., Muller, D. N., Luckow, B., Brocker, T., Debowski, K., Fritz, G., Opdenakker, G., Diefenbach, A., Biber, K., Heikenwalder, M., Geissmann, F., Rosenbauer, F., Prinz, M., 2013. Microglia emerge from erythromyeloid precursors via Pu.1- and Irf8-dependent pathways. Nat Neurosci $16,273-280$.

Kiguchi, N., Kobayashi, Y., Maeda, T., Saika, F., Kishioka, S., 2010a. CC-chemokine MIP-1alpha in the spinal cord contributes to nerve injury-induced neuropathic pain. Neurosci Lett 484, 17-21.

Kiguchi, N., Maeda, T., Kobayashi, Y., Fukazawa, Y., Kishioka, S., 2010b. Macrophage inflammatory protein-1alpha mediates the development of neuropathic pain following peripheral nerve injury through interleukin-1beta up-regulation. Pain 149, 305-315.

Kobayashi, K., Fukuoka, T., Yamanaka, H., Dai, Y., Obata, K., Tokunaga, A., Noguchi, K., 2006. Neurons and glial cells differentially express P2Y receptor mRNAs in the rat dorsal root ganglion and spinal cord. J Comp Neurol 498, 443-454.

Kobayashi, K., Takahashi, E., Miyagawa, Y., Yamanaka, H., Noguchi, K., 2011. Induction of the P2X7 receptor in spinal microglia in a neuropathic pain model. Neurosci Lett 504, 57-61.

Kobayashi, K., Yamanaka, H., Fukuoka, T., Dai, Y., Obata, K., Noguchi, K., 2008. $\mathrm{P} 2 \mathrm{Y} 12$ receptor upregulation in activated microglia is a gateway of p38 signaling and neuropathic pain. J Neurosci 28, 2892-2902.

Kurotaki, D., Osato, N., Nishiyama, A., Yamamoto, M., Ban, T., Sato, H., Nakabayashi, J., Umehara, M., Miyake, N., Matsumoto, N., Nakazawa, M., Ozato, K., Tamura, T., 2013. Essential role of the IRF8-KLF4 transcription factor cascade in murine monocyte differentiation. Blood 121, 1839-1849.

Liu, L., Rudin, M., Kozlova, E. N., 2000. Glial cell proliferation in the spinal cord after dorsal rhizotomy or sciatic nerve transection in the adult rat. Exp Brain Res 131, 64-73. Maeda, M., Tsuda, M., Tozaki-Saitoh, H., Inoue, K., Kiyama, H., 2010. Nerve injury-activated microglia engulf myelinated axons in a P2Y12 signaling-dependent manner in the dorsal horn. Glia 58, 1838-1846.

Mantyh, P. W., 2006. Cancer pain and its impact on diagnosis, survival and quality of 
life. Nat Rev Neurosci 7, 797-809.

Masuda, T., Iwamoto, S., Yoshinaga, R., Tozaki-Saitoh, H., Nishiyama, A., Mak, T. W., Tamura, T., Tsuda, M., Inoue, K., 2014. Transcription factor IRF5 drives P2X4R+-reactive microglia gating neuropathic pain. Nature Commun 5, 3771.

Masuda, T., Tsuda, M., Yoshinaga, R., Tozaki-Saitoh, H., Ozato, K., Tamura, T., Inoue, K., 2012. IRF8 is a critical transcription factor for transforming microglia into a reactive phenotype. Cell Rep 1, 334-340.

Matsushita, K., Tozaki-Saitoh, H., Kojima, C., Masuda, T., Tsuda, M., Inoue, K., Hoka, S., 2014. Chemokine (C-C motif) receptor 5 is an important pathological regulator in the development and maintenance of neuropathic pain. Anesthesiology 120, 1491-1503. McGaraughty, S., Chu, K. L., Namovic, M. T., Donnelly-Roberts, D. L., Harris, R. R., Zhang, X. F., Shieh, C. C., Wismer, C. T., Zhu, C. Z., Gauvin, D. M., Fabiyi, A. C., Honore, P., Gregg, R. J., Kort, M. E., Nelson, D. W., Carroll, W. A., Marsh, K., Faltynek, C. R., Jarvis, M. F., 2007. P2X7-related modulation of pathological nociception in rats. Neuroscience 146, 1817-1828.

Narita, M., Yoshida, T., Nakajima, M., Narita, M., Miyatake, M., Takagi, T., Yajima, Y., Suzuki, T., 2006. Direct evidence for spinal cord microglia in the development of a neuropathic pain-like state in mice. J Neurochem 97, 1337-1348.

Nimmerjahn, A., Kirchhoff, F., Helmchen, F., 2005. Resting microglial cells are highly dynamic surveillants of brain parenchyma in vivo. Science 308, 1314-1318.

Ochi-Ishi, R., Nagata, K., Inoue, T., Tozaki-Saitoh, H., Tsuda, M., Inoue, K., 2014. Involvement of the chemokine CCL3 and the purinoceptor P2X7 in the spinal cord in paclitaxel-induced mechanical allodynia. Molecular pain 10, 53.

Ohsawa, K., Irino, Y., Nakamura, Y., Akazawa, C., Inoue, K., Kohsaka, S., 2007. Involvement of $\mathrm{P} 2 \mathrm{X} 4$ and $\mathrm{P} 2 \mathrm{Y} 12$ receptors in ATP-induced microglial chemotaxis. Glia 55, 604-616.

Olechowski, C. J., Truong, J. J., Kerr, B. J., 2009. Neuropathic pain behaviours in a chronic-relapsing model of experimental autoimmune encephalomyelitis (EAE). Pain 141, 156-164.

Osterberg, A., Boivie, J., Thuomas, K. A., 2005. Central pain in multiple sclerosis--prevalence and clinical characteristics. European journal of pain 9, 531-542. Pausch, M. H., Lai, M., Tseng, E., Paulsen, J., Bates, B., Kwak, S., 2004. Functional expression of human and mouse P2Y12 receptors in Saccharomyces cerevisiae. Biochem Biophys Res Commun 324, 171-177.

Peters, C. M., Jimenez-Andrade, J. M., Jonas, B. M., Sevcik, M. A., Koewler, N. J., Ghilardi, J. R., Wong, G. Y., Mantyh, P. W., 2007. Intravenous paclitaxel administration 
in the rat induces a peripheral sensory neuropathy characterized by macrophage infiltration and injury to sensory neurons and their supporting cells. Exp Neurol 203, 42-54.

Pevida, M., Lastra, A., Hidalgo, A., Baamonde, A., Menendez, L., 2013. Spinal CCL2 and microglial activation are involved in paclitaxel-evoked cold hyperalgesia. Brain Res Bull 95, 21-27.

Qureshi, O. S., Paramasivam, A., Yu, J. C., Murrell-Lagnado, R. D., 2007. Regulation of P2X4 receptors by lysosomal targeting, glycan protection and exocytosis. J Cell Sci 120, 3838-3849.

Sasaki, Y., Hoshi, M., Akazawa, C., Nakamura, Y., Tsuzuki, H., Inoue, K., Kohsaka, S., 2003. Selective expression of Gi/o-coupled ATP receptor P2Y12 in microglia in rat brain. Glia 44, 242-250.

Scholz, J., Woolf, C. J., 2002. Can we conquer pain? Nat Neurosci 5, 1062-1067.

Schulz, C., Gomez Perdiguero, E., Chorro, L., Szabo-Rogers, H., Cagnard, N., Kierdorf, K., Prinz, M., Wu, B., Jacobsen, S. E., Pollard, J. W., Frampton, J., Liu, K. J.,

Geissmann, F., 2012. A lineage of myeloid cells independent of Myb and hematopoietic stem cells. Science 336, 86-90.

Shen, Y., Zhang, Z. J., Zhu, M. D., Jiang, B. C., Yang, T., Gao, Y. J., 2015. Exogenous induction of HO-1 alleviates vincristine-induced neuropathic pain by reducing spinal glial activation in mice. Neurobiology of disease 79, 100-110.

Shigemoto-Mogami, Y., Koizumi, S., Tsuda, M., Ohsawa, K., Kohsaka, S., Inoue, K., 2001. Mechanisms underlying extracellular ATP-evoked interleukin-6 release in mouse microglial cell line, MG-5. J Neurochem 78, 1339-1349.

Shiratori, M., Tozaki-Saitoh, H., Yoshitake, M., Tsuda, M., Inoue, K., 2010. P2X7 receptor activation induces CXCL2 production in microglia through NFAT and PKC/MAPK pathways. J Neurochem 114, 810-819.

Sorge, R. E., Mapplebeck, J. C., Rosen, S., Beggs, S., Taves, S., Alexander, J. K., Martin, L. J., Austin, J. S., Sotocinal, S. G., Chen, D., Yang, M., Shi, X. Q., Huang, H., Pillon, N. J., Bilan, P. J., Tu, Y., Klip, A., Ji, R. R., Zhang, J., Salter, M. W., Mogil, J. S., 2015. Different immune cells mediate mechanical pain hypersensitivity in male and female mice. Nat Neurosci.

Suter, M. R., Wen, Y. R., Decosterd, I., Ji, R. R., 2007. Do glial cells control pain? Neuron Glia Biol 3, 255-268.

Tamura, T., Yanai, H., Savitsky, D., Taniguchi, T., 2008. The IRF family transcription factors in immunity and oncogenesis. Ann Rev Immunol 26, 535-584.

Tanaka, T., Minami, M., Nakagawa, T., Satoh, M., 2004. Enhanced production of 
monocyte chemoattractant protein-1 in the dorsal root ganglia in a rat model of neuropathic pain: possible involvement in the development of neuropathic pain. Neurosci Res 48, 463-469.

Thacker, M. A., Clark, A. K., Bishop, T., Grist, J., Yip, P. K., Moon, L. D., Thompson, S. W., Marchand, F., McMahon, S. B., 2009. CCL2 is a key mediator of microglia activation in neuropathic pain states. European journal of pain 13, 263-272. Toulme, E., Garcia, A., Samways, D., Egan, T. M., Carson, M. J., Khakh, B. S., 2010. P2X4 receptors in activated C8-B4 cells of cerebellar microglial origin. J Gen Physiol 135, 333-353.

Toulme, E., Khakh, B. S., 2012. Imaging P2X4 receptor lateral mobility in microglia regulation by calcium and p38 MAPK. J Biol Chem 287, 14734-14748.

Toyomitsu, E., Tsuda, M., Yamashita, T., Tozaki-Saitoh, H., Tanaka, Y., Inoue, K., 2012. CCL2 promotes $\mathrm{P} 2 \mathrm{X} 4$ receptor trafficking to the cell surface of microglia. Purinergic Signal 8, 301-310.

Tozaki-Saitoh, H., Tsuda, M., Miyata, H., Ueda, K., Kohsaka, S., Inoue, K., 2008. $\mathrm{P} 2 \mathrm{Y} 12$ receptors in spinal microglia are required for neuropathic pain after peripheral nerve injury. J Neurosci 28, 4949-4956.

Trang, T., Beggs, S., Wan, X., Salter, M. W., 2009. P2X4-receptor-mediated synthesis and release of brain-derived neurotrophic factor in microglia is dependent on calcium and p38-mitogen-activated protein kinase activation. J Neurosci 29, 3518-3528. Tsuda, M., Beggs, S., Salter, M. W., Inoue, K., 2013a. Microglia and intractable chronic pain. Glia 61, 55-61.

Tsuda, M., Inoue, K., Salter, M. W., 2005. Neuropathic pain and spinal microglia: a big problem from molecules in "small" glia. Trends Neurosci 28, 101-107.

Tsuda, M., Kuboyama, K., Inoue, T., Nagata, K., Tozaki-Saitoh, H., Inoue, K., 2009a. Behavioral phenotypes of mice lacking purinergic $\mathrm{P} 2 \mathrm{X} 4$ receptors in acute and chronic pain assays. Molecular pain 5, 28.

Tsuda, M., Masuda, T., Tozaki-Saitoh, H., Inoue, K., 2013b. Microglial regulation of neuropathic pain. J Pharmacol Sci 121, 89-94.

Tsuda, M., Masuda, T., Tozaki-Saitoh, H., Inoue, K., 2013c. P2X4 receptors and neuropathic pain. Front Cell Neurosci 7, 191.

Tsuda, M., Mizokoshi, A., Shigemoto-Mogami, Y., Koizumi, S., Inoue, K., 2004. Activation of p38 mitogen-activated protein kinase in spinal hyperactive microglia contributes to pain hypersensitivity following peripheral nerve injury. Glia 45, 89-95. Tsuda, M., Shigemoto-Mogami, Y., Koizumi, S., Mizokoshi, A., Kohsaka, S., Salter, M. W., Inoue, K., 2003. P2X4 receptors induced in spinal microglia gate tactile allodynia 
after nerve injury. Nature 424, 778-783.

Tsuda, M., Toyomitsu, E., Komatsu, T., Masuda, T., Kunifusa, E., Nasu-Tada, K., Koizumi, S., Yamamoto, K., Ando, J., Inoue, K., 2008a. Fibronectin/integrin system is involved in $\mathrm{P} 2 \mathrm{X}(4)$ receptor upregulation in the spinal cord and neuropathic pain after nerve injury. Glia 56, 579-585.

Tsuda, M., Toyomitsu, E., Kometani, M., Tozaki-Saitoh, H., Inoue, K., 2009 b.

Mechanisms underlying fibronectin-induced upregulation of P2XR expression in microglia: distinct roles of PI3K-Akt and MEK-ERK signaling pathways. J Cell Mol Med 13, 3251-3259.

Tsuda, M., Tozaki-Saitoh, H., Masuda, T., Toyomitsu, E., Tezuka, T., Yamamoto, T., Inoue, K., 2008b. Lyn tyrosine kinase is required for P2X(4) receptor upregulation and neuropathic pain after peripheral nerve injury. Glia 56, 50-58.

Ulmann, L., Hatcher, J. P., Hughes, J. P., Chaumont, S., Green, P. J., Conquet, F., Buell, G. N., Reeve, A. J., Chessell, I. P., Rassendren, F., 2008. Up-regulation of P2X4 receptors in spinal microglia after peripheral nerve injury mediates BDNF release and neuropathic pain. J Neurosci 28, 11263-11268.

Vazquez-Villoldo, N., Domercq, M., Martin, A., Llop, J., Gomez-Vallejo, V., Matute, C., 2014. P2X4 receptors control the fate and survival of activated microglia. Glia 62, 171-184.

Wang, Y., Szretter, K. J., Vermi, W., Gilfillan, S., Rossini, C., Cella, M., Barrow, A. D., Diamond, M. S., Colonna, M., 2012. IL-34 is a tissue-restricted ligand of CSF1R required for the development of Langerhans cells and microglia. Nature immunology 13, 753-760.

White, F. A., Jung, H., Miller, R. J., 2007. Chemokines and the pathophysiology of neuropathic pain. Proc Natl Acad Sci U S A 104, 20151-20158.

Woolf, C. J., Mannion, R. J., 1999. Neuropathic pain: aetiology, symptoms, mechanisms, and management. Lancet 353, 1959-1964.

Woolf, C. J., Salter, M. W., 2000. Neuronal plasticity: increasing the gain in pain. Science 288, 1765-1769.

Yona, S., Kim, K. W., Wolf, Y., Mildner, A., Varol, D., Breker, M., Strauss-Ayali, D., Viukov, S., Guilliams, M., Misharin, A., Hume, D. A., Perlman, H., Malissen, B., Zelzer, E., Jung, S., 2013. Fate mapping reveals origins and dynamics of monocytes and tissue macrophages under homeostasis. Immunity 38, 79-91.

Zhang, J., De Koninck, Y., 2006. Spatial and temporal relationship between monocyte chemoattractant protein-1 expression and spinal glial activation following peripheral nerve injury. J Neurochem 97, 772-783. 
Zhang, J., Shi, X. Q., Echeverry, S., Mogil, J. S., De Koninck, Y., Rivest, S., 2007. Expression of CCR2 in both resident and bone marrow-derived microglia plays a critical role in neuropathic pain. J Neurosci 27, 12396-12406.

Zhang, Z., Zhang, Z. Y., Fauser, U., Schluesener, H. J., 2008. Mechanical allodynia and spinal up-regulation of $\mathrm{P} 2 \mathrm{X} 4$ receptor in experimental autoimmune neuritis rats.

Neuroscience 152, 495-501. 


\section{Figure Legend}

Figure 1 Schematic for the potential mechanisms by which purinergic signaling in activated microglia cause hyperexcitability in spinal dorsal horn neurons and neuropathic pain. Following peripheral nerve injury (PNI), activated microglia show increased IRF8 expression, which in turn leads to the induction of IRF5 expression. In response to fibronectin stimulation, IRF5 translocates to the nucleus and then induces $\mathrm{P} 2 \mathrm{X} 4 \mathrm{R}$ expression by directly binding to the promoter region of the P2rx4 gene. P2X4R protein localizes to intracellular lysosomes, and lysosomal exocytosis leads to P2X4R expression in the surface of microglia. P2X7R expression is also increased in spinal microglia after PNI. P2X4R and P2X7R are activated by extracellular ATP (which is presumably released from neurons or glial cells); this results in the subsequent release of bioactive diffusible factors, such as brain-derived neurotrophic factor (BDNF), proinflammatory cytokines and chemokines. BDNF downregulates the potassium-chloride transporter KCC2 through TrkB, causes an increase in intracellular $\left[\mathrm{Cl}^{-}\right]$, and leads to collapse of the transmembrane anion gradient in dorsal horn neurons, which in turn induces depolarization of these neurons after stimulation by $\gamma$-aminobutyric acid (GABA) and glycine. The resultant microglial-induced hyperexcitability in the dorsal horn pain network could be responsible for neuropathic pain. P2Y12R expression is also upregulated selectively in microglia after PNI. Engulfment of myelinated axons by activated microglia via P2Y12R signaling in the dorsal horn may also be involved in pathologically altered neuronal excitability in the dorsal horn and in abnormal pain. 


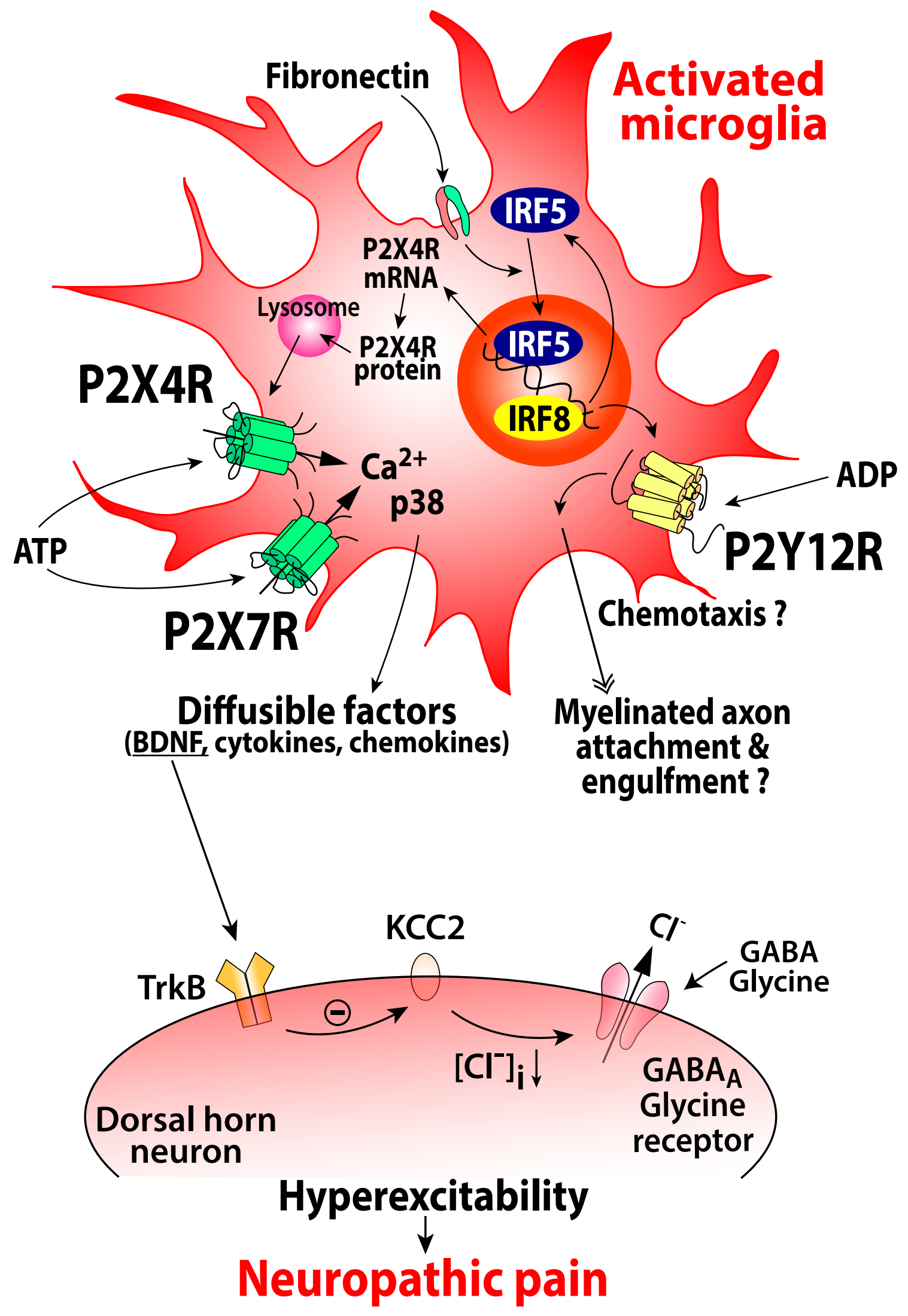

